\title{
LA ALFABETIZACIÓN DIGITAL EN LA FORMACIÓN DE COMPETENCIAS CIUDADANAS EN LA BÁSICA PRIMARIA*
}

\author{
DIGITAL LITERACY IN THE EDUCATION OF CITIZENSHIP COMPETENCES \\ IN ELEMENTARY SCHOOL
}

\author{
Gerzon Yair Calle-Álvarez** \\ ANGELINA LOZANO-PRADA***
}

\section{Resumen}

Objetivo. Describir las posibilidades que ofrece implementar una propuesta didáctica de alfabetización digital en la formación de competencias ciudadanas que den sentido a la pluralidad, identidad y valoración de las diferencias para estudiantes de quinto grado. Metodología. El método aplicado fue investigación-acción pues favorece el análisis de las acciones humanas en situaciones sociales reales; alcance descriptivo, con participación de 31 estudiantes del grado quinto de una institución educativa pública del departamento de Antioquia, Colombia, en la implementación de una secuencia didáctica orientada a fortalecer las competencias ciudadanas en un ambiente de aprendizaje apoyado por TIC; para la recolección de la información se aplicó el diario de campo. Resultados. Muestran que se requiere desarrollar las competencias instrumentales, cognitivas y sociales en una propuesta didáctica de alfabetización digital para la formación de competencias ciudadanas. Conclusiones. Un ambiente colaborativo mediado por TIC contribuye a la formación de competencias ciudadanas en la básica primaria.

Palabras clave: alfabetización digital, básica primaria, competencias ciudadanas, secuencia didáctica.

\begin{abstract}
Objective. To describe the possibilities offered by the implementation of a didactic proposal of digital literacy in the training in citizenship competences that give meaning to the plurality, identity and assessment of differences for fifth grade students. Methodology. The applied method was action research because it favors the analysis of human actions in real social situations. Descriptive scope, in which 31 fifth grade students of a public educational school of the department of Antioquia, Colombia, participated in the implementation of a didactic sequence aimed at strengthening citizenship competences in a learning environment supported by ICTs. For the gathering of information, the field diary was applied. Results. The results show that it is necessary to develop the instrumental, cognitive and social competences in a didactic proposal of digital literacy for the training in citizenship competences. Conclusions. A collaborative environment mediated by ICTs contributes to the training in citizenship competences in the elementary school.
\end{abstract}

Key words: digital literacy, elementary school, citizenship competences, didactic sequence.

\footnotetext{
${ }^{*}$ El presente artículo proviene de las reflexiones de la investigación "Alfabetización digital en la formación de competencias ciudadanas en la básica primaria”. Grupo de investigación: Didáctica y Nuevas Tecnologías, Universidad de Antioquia.

*** Universidad de Antioquia. Medellín, Colombia. E-mail: gerzon.calle@udea.edu.co

(D) orcid.org/0000-0002-4083-6051 Google Scholar

** Universidad de Antioquia. Medellín, Colombia. E-mail: angelina36107@gmail.com

(D) orcid.org/0000-0002-1511-0435 Google Scholar
} 
La alfabetización digital en la formación de competencias ciudadanas en la básica primaria

\section{Introducción}

El uso masivo de las tecnologías está marcando patrones de comportamiento social y configura una emergente cultura digital. Lo anterior, demanda del sector educativo replantear el currículo e incluir las tecnologías digitales. En este sentido, Gutiérrez y Tyner (2012) afirman que "la multiculturalidad, la digitalización de la información y el auge de las redes sociales, que son aspectos de la modernidad, no se abordan suficientemente desde la escuela" (p. 23). En consecuencia, la relación escuela y comunidad educativa se distancia al no encontrar espacios de concertación y diálogo en torno a la inclusión tecnológica. Entonces, la escuela se enfrenta al reto de incorporar las tecnologías al aula, para que profesores y estudiantes las vinculen a las actividades académicas y sus prácticas sociales.

Casas et al. (2015) afirman que "el ejercicio de la ciudadanía ha registrado importantes transformaciones en los últimos tiempos, ya que los ciudadanos se han volcado a distintos tipos de ejercicios y actividades ciudadanas, diferentes de las tradicionales, utilizando las TIC" (p. 222). Ante las formas de comunicación e interacción que ha adoptado la ciudadanía, resulta pertinente formar a los estudiantes en valores sociales a través de las competencias ciudadanas y digitales. Lo anterior, para que logren conocer, respetar, defender y promover los derechos fundamentales propios y ajenos en los actuales contextos digitales.

En la sociedad acceder al conocimiento aporta al progreso de los individuos y sus comunidades. De ahí que en la escuela se le dé importancia al qué, para qué y al cómo aprenden los estudiantes. Marzal (2009) afirma que "la Sociedad del Conocimiento precisa, en este contexto evolutivo, de una Sociedad del Aprendizaje, un aprendizaje especial y diferente, dada la distinta posición del educando, activo, autónomo y corresponsable en su proceso educativo" (p. 136). Es por eso que, en los nuevos modelos educativos se está enfocando la enseñanza hacia el desarrollo de competencias que les permitan a los estudiantes actuar y participar en los contextos digitalizados.

En la cultura digital, el ejercicio de la ciudadanía ha ganado espacios de expresión y comunicación, lo que ha implicado otras formas de alfabetización. La alfabetización digital y la formación ciudadana tienen una estrecha relación y un significado preponderante ante la masificación del uso de los dispositivos digitales. En consecuencia, el proceso de alfabetización implica la adquisición de competencias que faciliten la construcción de un sujeto que es capaz de interactuar a través de las tecnologías de la información y la comunicación (TIC), como ciudadano autónomo, crítico, reflexivo y con valores democráticos (Area, 2010). El presente artículo tiene como propósito presentar los resultados de una investigación que tenía en sus objetivos describir las posibilidades que ofrece la implementación de una propuesta didáctica de alfabetización digital en la formación de competencias ciudadanas que dé sentido a la pluralidad, identidad y valoración de las diferencias, en la dinámica curricular del área de ciencias sociales para la básica primaria. 


\section{Marco Teórico}

\section{Alfabetización digital}

Uno de los campos que más ha tenido transformaciones y a la vez ha ocasionado cambios en la sociedad del siglo XXI, es el de las tecnologías. De ahí que a la alfabetización digital se le esté dando atención en todos los ámbitos, especialmente en el educativo. Investigadores como Area (2010), Gross y Contreras (2006), Gutiérrez (2010), Pérez y Delgado (2012), Gutiérrez y Tyner (2012), Aguilar (2012), García, Ramírez y Rodríguez (2014) han investigado al respecto, con el fin de configurar un modelo integrado de teorización sobre el tipo de alfabetización que requieren los individuos en la era digital.

La cotidianidad gira en torno a lo digital, al punto que la gran mayoría de las actividades se realizan a través de algún dispositivo tecnológico. Y es que el mundo de hoy se caracteriza por ser digital y globalizado, donde la información y el conocimiento fluyen, circulan y son accesibles a todos en cualquier momento y lugar. Las anteriores características son propias de la emergente cultura digital. Una cultura donde la digitalización de la información que se encuentra en la red, se construye y se propaga rápidamente y está al alcance de todos. Gutiérrez y Tyner (2012) afirman que "la alfabetización para el siglo XXI, ha de ser mediática, digital, multimodal, critica y funcional” (p. 36). Desde esta perspectiva, la alfabetización es vista como una alfabetización multifuncional, cuyo fin ha de procurar una educación integral.

Aparte de que los estudiantes sean diestros en el manejo del software y el hardware y que desarrollen competencias cognitivas, también han de desarrollar actitudes y valores que orienten las acciones que realizan con las TIC, desde un sentido moral, ideológico y político (Area, 2010). En este punto, el autor converge con Gutiérrez y Tyner (2012) en la idea de no olvidar las actitudes y valores cuando se realizan actividades con las TIC. Por ello, la alfabetización digital se presenta como un instrumento formativo al servicio de una sociedad más justa, solidaria y democrática en la cultura digital (Travieso y Planella, 2008).

La investigación realizada por García et al. (2014) en el contexto escolar español, tenía como objetivo identificar los niveles de competencia mediática en niños y adolescentes de primaria y secundaria. A través de la aplicación de una metodología empírico-analítica fundamentada en la experimentación, con un posterior análisis estadístico, lograron identificar un bajo nivel en las competencias digitales en los estudiantes. Las autoras manifestaron que estos carecen de estas habilidades para ser considerados prosumidores digitales, por lo tanto, hay que plantear procesos de alfabetización que mejoren las competencias digitales en los estudiantes. En esta investigación se utilizó la encuesta con cuatro cuestionarios ad hoc online, los resultados dieron cuenta de que, aunque los jóvenes están en constante interacción con las TIC, carecen de las competencias digitales que les permita consumir y producir con estas. 
Para Gutiérrez (2003), el concepto de alfabetización digital consiste "en la capacidad de ser no solo receptor, también emisor en la era digital” (p. 4). Para el autor, estar alfabetizado digitalmente consiste en tener la capacidad no solo de obtener información, también analizarla, interpretarla y transmitirla. Por lo tanto, dependerá de la capacidad de comprensión y criticidad de lo leído por el usuario, lo que alcance a extraer y transmitir. En la perspectiva de Gross y Contreras (2006), consideran la alfabetización digital desde un sentido amplio, relacionado con las demandas de la sociedad actual.

\section{Competencias ciudadanas en la era digital}

Las competencias ciudadanas se convierten en el medio para generar espacios para la convivencia y el ejercicio de la democracia escolar que repercutan en mejores niveles de convivencia y, por ende, en mejores niveles académicos. Ruiz y Chaux (2005) definen las competencias ciudadanas como "el conjunto de capacidades y habilidades cognitivas, emocionales y comunicativas -integradas- relacionadas con conocimientos básicos (contenidos, procedimientos, mecanismos) que orientan moral y políticamente nuestra acción ciudadana” (p. 3). Es ahí, con el ejercicio autónomo y responsable de la acción ciudadana, donde se evidencia la adquisición de estas competencias.

Un reto en la acción educativa desde la escuela es desarrollar en los estudiantes las competencias para que participen en la construcción de ambientes escolares propicios para la interacción, la comunicación y el aprendizaje. En este sentido, el Ministerio de Educación de Colombia, MEN (2004) ha desarrollado el concepto de competencias ciudadanas desde varias perspectivas, una de ellas dice que son "el conjunto de conocimientos y habilidades cognitivas, emocionales y comunicativas que, articulados entre sí, hacen posible que el ciudadano actúe de manera constructiva en la sociedad democrática" (p. 8).

Desde la escuela hay que pensar en las competencias ciudadanas para la formación de personas solidarias, empáticas, que respeten y hagan respetar los derechos humanos propios y ajenos. Personas que en su actuar tomen decisiones teniendo en cuenta su bienestar y el de los demás, que estén dispuestos a trabajar en grupo con metas y objetivos comunes. Un ciudadano del siglo XXI tiene una manera de pensar orientada a la construcción de democracia participativa e incluyente, asume una concepción de ciudadanía no solo local, también global. Por lo anterior es que, desde la escuela, a los estudiantes se les ha de orientar para que ejerzan una ciudadanía activa.

Malfasis (2014) llevó a cabo una investigación en la Institución Educativa Diversificado de Chía, sobre el fortalecimiento de competencias ciudadanas a través de estrategias mediadas por las TIC. Su objetivo principal estaba enfocado en analizar la influencia de estrategias mediadas por las TIC en el fortalecimiento de la competencia ciudadana de convivencia y paz, desde la 
base del respeto en los estudiantes de básica primaria. El estudio se desarrolló en el curso de $3^{\circ} \mathrm{B}$ de la básica primaria, conformado por 19 niñas y 15 niños con edades entre 8 y 11 años. Se empleó el método de teoría fundamentada con un enfoque mixto en el cual predominó el de corte cualitativo.

Con respecto a la competencia ciudadana, Malfasis (2014) considera que para fortalecer estas competencias se ha de llevar a cabo un proceso de formación ciudadana basado en el respeto, mediante estrategias que propicien un ambiente donde la convivencia y resolución de conflictos se dé pacíficamente. También en la competencia, convivencia y paz concluye que se mostró una evolución satisfactoria ya que los estudiantes relacionaban la acción de vivir en comunidad con la ausencia de conflictos acudiendo al ejercicio del buen diálogo. Otra conclusión es que fue evidente la disminución de los conflictos, las respuestas agresivas y las quejas de los estudiantes en clase.

Formar para la ciudadanía es una tarea mancomunada entre familia y escuela, es una labor que compromete a toda la sociedad. Lo anterior reconoce que el sujeto aprende desde cualquier instancia a través de los medios de comunicación, la música, en el parque, con la televisión, con los diversos comportamientos ciudadanos desde lo político, cultural y social. Es por esto que la escuela, con miras de contribuir a la formación ciudadana, viene buscando desarrollar en los estudiantes la capacidad de ser sujetos autónomos, activos y críticos de las realidades en los diferentes contextos donde interactúe.

\section{Metodología}

La investigación educativa es una práctica que favorece un análisis concienzudo de esas situaciones relevantes que inquietan a los profesores. Amaya (2007) afirma que "la investigación educativa consiste en analizar con rigurosidad y objetividad una situación educativa entendida en sentido amplio" (p. 17). Para este estudio, se optó por el enfoque cualitativo al considerar que por su carácter inductivo permitía explorar y describir elementos propios del planteamiento del problema para luego generar otras perspectivas teóricas (Hernández, Fernández y Baptista, 2010). Esta característica permitió al investigador entrar al campo, observar y describir aquello que le causaba inquietud, para luego darle una interpretación desde un sentido crítico reflexivo, generando así otros discursos. Así mismo, se consideró el enfoque cualitativo apropiado para dar respuesta a componentes de la pregunta de investigación, la cual indaga por el cómo a través de una propuesta de alfabetización digital orientada a la formación de competencias ciudadanas otorga sentido a la pluralidad, identidad y valoración de las diferencias.

En cuanto al método aplicado en esta investigación, se tuvo en cuenta la investigaciónacción porque favorece el análisis de las acciones humanas en situaciones sociales reales. Para Elliot (2003): "La investigación-acción se relaciona con problemas prácticos cotidianos 
experimentados por los profesores, en vez de los problemas teóricos” (p. 24). La investigaciónacción permite profundizar en la comprensión de las distintas situaciones que se presentan durante la práctica pedagógica a través de la observación, el análisis y la interpretación, llegando a autorreflexionar sobre dichas situaciones. Es así como para la investigación este método fue relevante, al permitir recoger las percepciones de los implicados en el problema o situación desde un lenguaje propio, sencillo y habitual, logrando describir y explicar las acciones tal y como las vivenciaban los estudiantes, en su cotidianidad, en el ámbito escolar.

El alcance de esta investigación fue de carácter descriptivo, obteniendo los resultados a partir del análisis de la información arrojada en los diferentes instrumentos. Hernández et al. (2010) afirman que este alcance "busca especificar las propiedades, las características y los perfiles de personas, grupos, comunidades, procesos, objetos o cualquier otro fenómeno que se someta a un análisis” (p. 80). Es decir, la descripción se convierte en herramienta fundamental para localizar esos factores que inciden en el problema planteado. Para llevar a cabo este proceso de investigación se trazaron las siguientes fases: a) socialización del proyecto a la comunidad educativa; b) tanteo al estado actual del objeto de estudio; c) implementación de la propuesta didáctica e instrumentos desde la observación, acción y la reflexión; d) evaluación de la propuesta didáctica; e) análisis, reflexión e interpretación de la información.

Esta investigación se llevó a cabo en la Institución Educativa Luis Eduardo Díaz, zona urbana del municipio de Yondó, Antioquia, con uno de los seis grupos que conformaban el grado $5^{\circ}$. Este grupo estuvo conformado por 10 mujeres y 21 hombres entre las edades de 9 y 12 años, pertenecientes a los estratos socioeconómicos 1 y 2. La implementación del estudio se llevó a cabo durante los periodos de clases semanales de las áreas de ciencias sociales, ética y valores y tecnología e informática, durante el primer periodo del año lectivo 2017. Las sesiones de trabajo se desarrollaron así: en el aula de clase un 20\%, en la explicación de la teoría y actividades lúdicas, y en el aula de informática el 80\% restante en las actividades, donde era necesario el uso de recursos digitales.

Para el trabajo de aula se planeó una secuencia didáctica en la cual se procuró la integración curricular de áreas como ciencias sociales, ética y valores, y tecnología e informática. El desarrollo de ésta se realizó en ocho semanas y en periodos de ocho horas, tiempo durante el cual los estudiantes realizaron las actividades planeadas en el aula de informática. La secuencia didáctica se desarrolló en nueve sesiones y cada sesión estaba compuesta por cinco momentos: exploración de saberes previos, estructuración, actividad práctica, socialización y actividad formativa, y donde el producto final era la construcción colectiva de una revista digital. Los temas y actividades fueron pensados teniendo en cuenta los objetivos a alcanzar con la investigación. Se consideró la secuencia didáctica como estrategia metodológica, ya que, por su forma de organización jerárquica y secuencial de contenidos, permite generar experiencias cognitivas y conductuales en los estudiantes, integrando el trabajo individual con el grupal y 
colaborativo realizado dentro y fuera del aula con ayuda del profesor (Tapia, Correa, Ortiz y Neira, 2012). Durante el proceso se iba tomando nota de lo acontecido en el aula para luego registrarlo en el diario de campo.

El diario de campo es una herramienta que permite un registro detallado de experiencias significativas que suceden durante el ejercicio educativo en el aula de clase: "Es una narración detallada y periódica de experiencias vividas y de hechos observados por el investigador" (Cerda, 1991, p. 249); que se van registrando en una libreta de notas para luego organizar, analizar e interpretar. Aplicar el diario de campo como técnica de recolección de datos permitió sistematizar la práctica y experiencia investigativa, desde la percepción e interpretación de la realidad escolar, permitiendo al profesor cuestionarse sobre la práctica educativa. Este instrumento, al permitir reflexionar sobre lo que sucede en el aula desde una postura pedagógica, le ofrece insumos al profesor para que emprenda acciones en función del mejoramiento de su práctica educativa.

El diario de campo aplicado en esta investigación estuvo estructurado teniendo en cuenta las categorías sobre las cuales se basó la investigación como fueron: alfabetización digital, competencias ciudadanas y valoración de las diferencias, unas preguntas orientadoras que permitieron recoger información relevante, analizada luego desde una postura de observación y descripción objetiva, llegando a una reflexión pedagógica del investigador. En esta, se narraron hechos relevantes observados en los estudiantes durante las clases, relacionados con los comportamientos y actitudes con respecto a sus formas de pensar, de asociación e interacción, según las individualidades y creencias de cada uno. A partir de la observación participativa y por medio de preguntas orientadoras que permitieron una descripción de lo observado, se complementó con una reflexión pedagógica para interpretar y dar sentido a las actuaciones de los estudiantes en las diferentes sesiones y actividades planteadas. En la recolección de la información con este instrumento, primero se tomaron apuntes de lo observado en un cuaderno como notas de campo, tratando de recoger expresiones, palabras, gestos y demás comentarios que tuvieran resonancia o indicaran algo significativo con respecto al objeto de estudio.

El método utilizado para este análisis de la información fue el análisis de contenido, el cual consiste en la lectura e interpretación de contenidos sobre todo en documentos escritos (Ruiz, 2012). Con este método, a través de la lectura se extrajo información de las ideas expresadas por los participantes, las cuales habían quedado plasmadas en el diario de campo. El análisis de los datos a través de este método permitió hacer inferencias de gran valor en las diferentes transcripciones. Ruiz (2012) afirma que "la inferencia es elemento central del análisis de contenido” (p. 195). De ahí que, la aplicación de este método en el análisis fuera adecuada, ya que a partir de la lectura de los textos transcritos, se alcanzó a realizar interpretaciones relacionadas con los elementos que componen la pregunta y el objetivo de investigación. 
La alfabetización digital en la formación de competencias ciudadanas en la básica primaria

\section{Resultados y discusión}

El diario de campo permite registrar de manera pormenorizada una gran cantidad de variados datos. En el aula permite un registro detallado de experiencias significativas que suceden durante la práctica educativa. Este instrumento resulta para el investigador una gran herramienta auto-reflexiva que le permite plasmar su sentir, desde un punto de vista subjetivo, objetivo y pedagógico. Para el análisis de la información arrojada por el diario de campo, se utilizó un cuadro de doble entrada compuesto por filas y columnas, el cual permitió organizar y comparar la información de los datos que se cruzaban. Utilizar esta matriz facilitó el análisis alterno de los dos factores (observación: descripción objetiva y la reflexión pedagógica) tenidos en cuenta en el instrumento.

Indagar el nivel de adquisición de las competencias digitales en los estudiantes, resultaba indispensable para tener claridad sobre cuáles eran las habilidades que poseía cada estudiante. En la medida que se realizaba el análisis fueron emergiendo algunas subcategorías como: la competencia instrumental, la competencia cognitiva, la competencia emocional y las competencias sociales y ciudadanas. En cuanto a la competencia instrumental, relacionada con el uso del computador, se observó en la mayoría de los estudiantes que presentan dificultades en el manejo de algunas funciones básicas del software, como abrir y utilizar herramientas como Microsoft Word, Microsoft PowerPoint, resaltar, guardar textos, abrir una carpeta nueva. Así mismo, se observó que al escribir con el teclado, lo hacían con mucha lentitud y solo con un dedo. En la medida que se iba avanzando se apropiaban más del recurso digital, viéndoseles trabajar día a día con más independencia. De esta manera, se pudo evidenciar en el estudiante un aprendizaje progresivo en cuanto a la adquisición de las competencias instrumentales, que cada día iban mejorando y resolviendo las dificultades que tuvieron al principio siendo cada vez más independientes en el uso.

En cuanto a la competencia cognitiva relacionada con la búsqueda, selección, análisis e interpretación de la información (Area, 2010), los estudiantes presentaron algunas dificultades en las primeras clases, algunos no sabían cuál era el editor de texto, así como qué palabras o frases escribir, por lo que fueron varias las preguntas que hicieron como: ¿Profesor, dónde busco? ¿Profesor, qué palabras escribo? Aunque los estudiantes estaban en quinto grado, había algunos más aventajados que otros en cuanto a la adquisición de las competencias digitales. La mayoría de los estudiantes de la institución habitaban en barrios de estrato uno, carentes de los servicios básicos y desde luego de internet y computadores, por lo que las oportunidades de interactuar con las TIC, se limitaban a la escuela y a la clase de tecnología e informática. Estos estudiantes están en desventaja con los otros niños que viven en los barrios centrales, pues estos lograban acceder a internet desde varios espacios (casa, internet al parque, biblioteca, punto digital, café internet y servicios móviles). 
Al interactuar con las TIC, los estudiantes no solo ponen a prueba las competencias instrumental y cognitiva, también están presentes las competencia comunicativas, emocionales y sociales, las cuales están relacionadas con las competencias digitales. De ahí que el desarrollo de éstas sea importante para el estudiante, ya que le pueden permitir una actitud social positiva hacia los demás, significativa para el trabajo colaborativo, el respeto y la empatía en redes sociales (Area, 2010). Aprender a tener control de las emociones le repercutía al estudiante tener unas buenas relaciones sociales en los contextos análogos y digitales.

Cuando el estudiante buscaba un tema o asunto de su interés en internet, llegaba a sentirse abrumado por la cantidad de información que este le arroja. Durante la secuencia se observó que los estudiantes al buscar información en la web, son indecisos y se inquietan por la cantidad de enlaces que aparecen. En este aspecto, este instrumento arrojó las siguientes subcategorías: competencia cognitiva, competencia instrumental, información en la web. La competencia cognitiva y la instrumental relacionadas con la búsqueda y selección de información se manifestaron durante todo el proceso. De entrada, los estudiantes tuvieron dificultades con la búsqueda ya que cuando se les pidió que buscaran información acerca del trabajo colaborativo, primer tema que se trabajó, algunos estudiantes no lo hicieron, cuando se les preguntó el ¿por qué no lo habían hecho?, una estudiante contestó "profesora, es que no sé qué escribir", otra dijo, "profesora, qué escribo para que me salga". En la medida que se les fue explicando cómo hallar información les fue más fácil la búsqueda.

En cuanto a la comprensión y organización de la información que encontraban en internet, cuando escribían en Google, que fue el buscador más utilizado, este les arrojaba los diferentes enlaces donde hallar la información, los estudiantes tendían a abrir el primer enlace que aparecía y cuando eran lecturas extensas las desechaban. Para seleccionar los resultados de las búsquedas se identificó en los estudiantes algunas dificultades ante la cantidad de información que arroja la web. Al pedirles que leyeran e hicieran algún resumen, a algunos no les gustaba, otros estudiantes cortaban y pegaban alguna información, aunque a veces irrelevantes o pegaban todo el texto sin resumir.

Durante las clases se pudo evidenciar que a los estudiantes se les dificulta comprender y organizar la información que encuentran en la web, ya que ante las actividades que implicaban lectura de grafías, la dejaban a un lado inclinándose hacia otras actividades como juegos online, videos, Facebook, o buscar y oír música. En cuanto a la competencia cognitiva, el MEN (2004) la define como la capacidad para realizar diversos procesos mentales, fundamentales en el ejercicio ciudadano. El desarrollo de las competencias cognitivas y las instrumentales le ha de brindar al individuo un mayor desarrollo y capacidad de análisis, comprensión y organización de la información y difusión de contenidos apropiados. 
La alfabetización digital en la formación de competencias ciudadanas en la básica primaria

La integración de las TIC en las clases por parte de la docente fue en aumento, al igual que en los estudiantes. Evidenciar la forma como los estudiantes integraban los recursos TIC (aplicaciones, videos, procesadores de textos, audios) a su proceso educativo sirvió para una claridad en cuanto a los presaberes que tenía cada uno. Aunque los estudiantes presentaron dificultades con algunas funciones de Microsoft Word y la búsqueda de la información, durante el desarrollo de la secuencia didáctica mostraban interés por las actividades que se les planteaban. Durante las sesiones el trabajo colaborativo para construcción de la revista digital fue protagonista, a través de él, los estudiantes demostraron poseer buenos valores como el compañerismo y la ayuda mutua, cuando alguien tenía alguna dificultad había alguien dispuesto a colaborar. Onrubia, Colomina y Engel (2008) basados en las perspectivas cognitivas de Piaget y la perspectiva sociocultural de Vygotsky, establecen que el aprendizaje colaborativo se conceptualiza como un proceso de interacción conjunta donde se comparte, se negocia y se construyen significados para solucionar problemas, crear o producir algo. Desde esta mirada, el trabajo colaborativo que se produjo en el aula durante las distintas sesiones tuvo relación con esta definición, ya que cuando alguno de los estudiantes tenía algún problema, alguien estaba dispuesto a colaborarle.

Trabajar en grupo con recursos TIC les facilitó solucionar las dificultades que se presentaron, cuando alguno no sabía o no entendía como trabajar con alguna aplicación, otro compañero más diestro ayudaba a solucionar las dificultades que presentaba. Otro aspecto a destacar es la actitud que mostraban, lo motivados que se veían para ir al aula de informática y el interés por la actividad, preguntaban cuándo no entendían. De igual forma, el establecimiento de normas para el trabajo colaborativo en el aula fue de gran ayuda ya que permitió más organización y entendimiento entre todos.

Para los estudiantes trabajar en el computador les resultó motivador, pues siempre mostraban buena actitud y aunque algunos todavía no contaban con las habilidades suficientes, hacían el intento. Que el estudiante desarrollara las competencias emocionales e instrumentales, le brindaba seguridad cuando interactúa con las TIC. En la medida que se ejecutaba el trabajo en el aula durante la secuencia didáctica, se pudo evidenciar que entre más contacto tenían los estudiantes con el computador, más se afianzaban en la búsqueda de información o al realizar otras actividades.

En la interacción diaria que se daba entre los estudiantes en el aula de clase, se veían enfrentados a situaciones donde se dejaban entrever los valores que poseían. Durante el desarrollo de las sesiones de la secuencia didáctica realizando actividades para la revista digital, fue posible observar en algunos estudiantes la vivencia de los valores sociales en sus comportamientos. Al inicio de la primera sesión los estudiantes que tuvieron que compartir computador se mostraron inconformes. Esta actitud fue mejorando en la medida que iba pasando el tiempo en el aula interactuando con las TIC. El que se haya establecido unos acuerdos de convivencia favoreció el trabajo en equipo, el aprendizaje colaborativo y el clima escolar. 
Como la mayoría de las actividades implicaron trabajar en grupo, se fomentó el aprendizaje colaborativo y el fortalecimiento de valores como el compañerismo, la tolerancia, el respeto y la ayuda mutua evidenciados en los comportamientos de los estudiantes. Avello et al. (2013) afirman que "el aprendizaje con los otros favorece la formación de habilidades y valores" (p. 453). De esta manera, el aprendizaje colaborativo fue un aspecto que estuvo presente durante las diferentes sesiones de la secuencia didáctica.

En general, los estudiantes adoptaron buenos comportamientos cuando se trabajó la secuencia didáctica, ya fuese viendo un video o realizando otra actividad con o sin internet. Las dificultades que se presentaron al principio disminuyeron en la medida que se avanzaba e interactuaban entre ellos y con las TIC. Solían ser colaboradores y compartían las actividades que realizaban, mostrándolas a sus compañeros como buscando una opinión o simplemente un me gusta. La existencia de unos acuerdos visibles en el aula ayudó a regular los comportamientos en los estudiantes.

Evidenciar la manera como manifestaban la vivencia de las competencias ciudadanas, pluralidad, identidad y valoración de las diferencias durante las clases que implicaban el uso de las TIC, era relevante para el desarrollo de los objetivos de este estudio. Durante las primeras clases de la secuencia didáctica se pudo entrever el individualismo en los estudiantes, ellos preferían trabajar solos, manifestando que así trabajaban mejor, por eso cuando les tocaba compartir el computador con otro compañero hacían gestos de desagrado, a no ser que les tocara con alguien de sus afectos. En una de las clases un estudiante dijo la siguiente expresión "profe, déjeme hacer en un computador solo, es que esos niños son muy envidiosos y no lo dejan trabajar a uno". Aunque en el grupo solo hay un estudiante de descendencia afro lo aceptaban y trabajaban con él, pero a veces cuando se enojaban le decían frases despectivas como "negro ese”.

En ocasiones los estudiantes no se miden cuando dicen cosas para referirse a algún compañero y llegan a dar trato discriminador. En el contexto escolar este tipo de expresiones son frecuentes, ante esto, los estudiantes deben aprender desde pequeños que ningún maltrato o forma de discriminación es justificable; aunque para algunas comunidades o grupo social esto lo puedan considerar como una práctica educativa aceptable (Ruiz y Chaux, 2005). Crear conciencia en el estudiante, sobre el respeto y la valoración de las características y valores que poseen sus compañeros de clase, es parte de la tarea del profesor y se trasmite a veces a través del currículo oculto.

El grupo en general se mostró receptivo cuando se trataba de acoger a otros, igualmente cuando trabajaban con alguien que tenía dificultades con las actividades, ellos se ayudan. En la medida que realizaban actividades en grupo, los comportamientos de los estudiantes mejoraban al punto que no importaban con quien lo hacían, se ayudaban mutuamente sin 
La alfabetización digital en la formación de competencias ciudadanas en la básica primaria

reparo. Esto permitió observar la valoración del saber en sus compañeros, pues cuando alguien tenía alguna dificultad los estudiantes buscaban ayuda sin importar quién era, lo que importaba era que les pudieran resolver la dificultad. De esta manera, se logró evidenciar que la vivencia de las competencias pluralidad, identidad y valoración de las diferencias por los estudiantes se da de manera normal y natural cuando realizaban actividades apoyados por TIC.

La vivencia de los valores (tolerancia, respeto a las diferencias) que sustentan una sana convivencia en la multiculturalidad del grupo de estudiantes, fortalecieron la unidad en el grupo. Por lo general, cuando los estudiantes están entre el grupo de amigos son espontáneos en su forma de comunicarse, inventan juegos bruscos y adoptan un vocabulario y unas expresiones un tanto groseras para distraerse, cosa que para ellos es algo normal, así lo manifestaban. Entonces surgen las burlas, los apodos, los tratos despectivos, que dado el momento son generadores de conflictos, cuando alguno no está de buen humor como para aguantar esos tratos. Lo anterior se vio reflejado durante la secuencia en las primeras clases, presentándose algunos inconvenientes entre compañeros debido a esos comportamientos, ante esto se tuvo que recordar constantemente los pactos de convivencia construidos colectivamente y el propósito de ellos.

La vivencia de los valores como la tolerancia y el respeto por el otro dentro del grupo reflejó algunas falencias que ameritaron conversar y reflexionar sobre estos valores y la importancia de la comunicación asertiva y efectiva. Cabe destacar que el grupo es diverso, compuesto por estudiantes que proceden de distintas regiones, principalmente de los departamentos de Santander, Antioquia y Bolívar, por lo que al interactuar dentro o fuera del aula había manifestaciones de diversos actos y costumbres variadas. Es por esto que, en un principio, la práctica de los valores se vio matizado por las costumbres y significados propios de cada quien y que en algunos momentos llegaron a alterar el orden y la convivencia. Sin embargo, el haber trabajado en grupo usando recursos TIC, temas como los valores para la convivencia, la discriminación y el ciberbullying, pluralidad, identidad y las diferencias para la revista digital, generó gran impacto en los estudiantes, mejorando los comportamientos y logrando que valoraran las diferencias de cada quien.

Los estudiantes interactuaban constantemente con sus compañeros en el aula de clase, donde a través del acto comunicativo reflejaban sus fortalezas o debilidades en cuanto a la sociabilidad. Es ahí cuando los conocimientos, las habilidades que poseen junto con las actitudes que adoptan tienen sentido, si se utilizan en la comprensión y resolución de los problemas a través de la reflexión y la acción, es decir, hacen uso de las competencias sociales y ciudadanas que poseen (Pagés, 2012). Durante el desarrollo de la secuencia didáctica se alcanzó a generar una conciencia colectiva sobre la importancia de la práctica de los valores, como la tolerancia, el respeto, logrando generar empatía y aceptación entre los estudiantes, lo cual fue significativo para lograr una convivencia basada en el respeto a las diferencias, modos de pensar, actuar o de sentir. Para 
esto fue primordial trabajar en la comunicación asertiva, permitiendo que los estudiantes se expresaran de manera clara, sin temor a ser juzgados o recriminados por lo que decían.

En las distintas sesiones, cuando se realizaban las actividades para la revista, se pudo observar la motivación que generaba en los estudiantes el uso de los recursos digitales en las clases. De ahí que la motivación y la expectativa que se generaron desde el primer día en los estudiantes fueron constantes durante la secuencia didáctica. Siempre estaban atentos a las horas indicadas en el horario de clase y preguntaban sobre las actividades a desarrollar. Preguntas como: "Profesor, qué vamos a realizar en la clase hoy" o "profesor, yo le ayudo a instalar los computadores hoy" se escuchaban con frecuencia. Para los estudiantes ir al aula de informática y trabajar con las TIC les resultaba motivador, esto se corroboraba cuando manifestaban que les gustaba ir allí, porque era cambiar de ambiente y aprender cosas nuevas y de modo diferente.

El uso del computador durante las clases fue significativo para los estudiantes, sobre todo para aquellos que la única oportunidad que tenían de interactuar con ellos era en la hora de tecnología e informática. Aunque algunos se mostraban tímidos con el computador ya que desconocían muchas funciones del software, hacían el intento por realizar las actividades, preguntaban a algún compañero o llamaban a la profesora para que les colaborara cuando no entendían algo; además, durante todas las sesiones demostraron agrado y satisfacción de estar trabajando en el aula de informática. De otro lado, fue motivante ver a los estudiantes atentos desarrollando las actividades propuestas para la revista y dispuestos a colaborarle a los que tenían dificultades. Otro aspecto que llamó la atención fue ver la atracción que ejerció el computador en algunos estudiantes totalmente absortos; de ahí que podía llegar a pensar que entre estudiante y computador resulta una relación directa de compenetración que lleva al estudiante como a desconectarse de la realidad.

Vincular los recursos TIC a los procesos educativos es una tarea que se ha venido dando gradualmente en la escuela mediante estrategias de enseñanzas y aprendizaje, y a lo cual apuntan los objetivos de este trabajo investigativo. Evidenciar la formación de competencias ciudadanas en los estudiantes cuando realizaban actividades que implican el uso de recursos TIC, se logró observando sus comportamientos durante las clases. En las primeras sesiones de la secuencia, al llegar al aula se generaba desorden, pues todos querían entrar de primeros al aula. Tratando de organizar la entrada, se les pidió hacer fila, entrar de a uno e irse ubicando en orden; seguidamente se recordaban las normas que se habían construido para ponerlas en práctica, esto funcionó así durante toda la secuencia, de esta manera se evitaron los roces en la entrada y dentro de ella. Ya ubicados cada uno en su silla surgió otra situación poco satisfactoria y fue que como había 20 computadores de los cuales solo 18 servían, debían compartirlos, esto generó malestar en algunos estudiantes que querían trabajar solos. En las primeras clases se vieron caras de insatisfacción, ya después se fueron acomodando y aceptando compartir el computador con algún compañero. 
La situación anterior favoreció las relaciones interpersonales entre los compañeros, a medida que se trabajaba en el aula y en grupo las dificultades fueron disminuyendo. Se les veía conversar entre ellos, si no estaban de acuerdo en algo pedían la opinión de la profesora u otro compañero. Aunque había estudiantes que no les gustaba que los molestaran, había otros que sí colaboraban con gusto, como el caso de "Juan Camilo" un estudiante aventajado en el uso del computador que siempre estaba dispuesto a colaborar sin importar si era niña o niño, o si sabía poco o no. Juan Camilo fue un ejemplo claro del cambio de actitud durante las clases en el aula de informática, pues normalmente en clase le cuesta mucho adoptar una actitud de escucha y buen comportamiento, por lo que frecuentemente se le está pidiendo que por favor colabore con el orden. Contrario a esto, la actitud de Juan Camilo en el aula de informática, cuando estaba frente al computador realizando alguna actividad, fue de amabilidad y colaboración, allí poco se le llamó la atención.

Lo anterior indica que, desde la didáctica de las ciencias sociales, conformar una propuesta didáctica para la formación de competencias ciudadanas apoyadas por TIC, repercutirá en los comportamientos sociales de los estudiantes dentro del aula. Siendo así, usar los recursos TIC en estrategias para la formación en competencias ciudadanas debe arrojar buenos resultados si se planifican, brindándole una participación real al estudiante para que desarrolle la autonomía. Montaño (2015) afirma que "la educación en ciudadanía y valores, la formación ciudadana, debe contar con la práctica real, es decir, contar con una participación activa de los individuos” (p. 89). La tarea de crear ambientes de aprendizajes mediados por las TIC, desde la integración de áreas, en este caso ciencias sociales, ética y valores, y tecnología e informática, es responsabilidad no solo del profesor, sino también del equipo administrativo, quienes han de velar porque haya los recursos, los espacios adecuados y la red de internet a disposición de profesores y estudiantes.

Con la aplicación de las estrategias planteadas en la secuencia didáctica usando herramientas de TIC, y desde la didáctica de las ciencias sociales, se esperaba que arrojaran unos resultados satisfactorios con respecto a la construcción de ciudadanía. Debido a que al principio de la secuencia había estudiantes poco habilidosos en el manejo de computador, el desarrollo de las actividades fue lento. Sin embargo, en la medida que se frecuentaba el aula de informática se volvían más diestros. El haber usado aplicaciones de la web, aparte de haber sido atractivo para los estudiantes, permitió desarrollar la creatividad e incentivar el aprendizaje colaborativo, pues algunos se vieron obligados a buscar la ayuda de algún compañero cuando tenían dificultades.

Durante la aplicación de la secuencia didáctica, se observó cómo los comportamientos de los estudiantes se modificaron considerablemente. Es el caso de Nilton, estudiante que normalmente era agresivo con sus compañeros, en un principio fue un poco intolerante en el aula de informática, pero con el transcurrir de las actividades se le vio más controlado. Debido a su fuerte temperamento, se impacientaba un poco cuando no le salían las cosas bien, entonces buscaba la ayuda de algún compañero. 
Durante el trabajo se observó que los estudiantes contribuían cada uno desde su saber en la realización de la actividad, de esta manera, lo que producían era producto de los dos o tres, esto les permitió valorar el saber en sus compañeros y comprender la importancia del trabajo colaborativo. Onrubia et al. (2008) afirman que, "en la colaboración, cada miembro del grupo contribuye a la resolución conjunta del problema; la colaboración depende, por ello, del establecimiento de un lenguaje y significados comunes respecto a la tarea, y de una meta común al conjunto de participantes” (p. 235). Aprovechar estas posibilidades que ofrece la web de interacción y construcción colaborativa del conocimiento, procurando su uso pedagógico en el aula, puede llegar a ser una estrategia de gran significado para fomentar el aprendizaje colaborativo y, por ende, la construcción de valores y principios ciudadanos si se planifica desde los planes de área. Haber utilizado recursos TIC como aplicaciones para hacer caricatura, sopa de letras, historietas, collages, fue gratificante para los estudiantes y les permitió ser creativos, sociables, participativos y críticos de sus producciones y las ajenas.

Evaluar los procesos formativos permite reflexionar sobre los resultados y mejorar las estrategias que se aplican con el fin de que estas redunden en mejores aprendizajes. Para esto la evaluación formativa es una herramienta que favorece revisar los resultados para mejorar las enseñanzas y por derecha los aprendizajes en los estudiantes (Popham, 2013). Como en todo proceso educativo, es importante identificar las fortalezas y debilidades y la calidad de los aprendizajes que fructificaron con la aplicación de las actividades programadas con el fin de realizar acciones de mejoramiento. En la medida en que los estudiantes iban interactuando con las TIC, se pudo ir evidenciando su progreso en cuanto a su desempeño. Cada estudiante presentó un rendimiento diferente de acuerdo con sus conocimientos, es así como la aplicación de evaluación formativa durante el proceso requirió estar al tanto del desenvolvimiento de cada estudiante durante la actividad para no perder el hilo del desarrollo de cada proceso. Cada estudiante presentó una forma de trabajar y resolver las dificultades que se presentaban con las TIC, de manera diferente, por lo tanto, a través de la observación del desarrollo de las actividades y del progreso que los estudiantes demostraban, se logró verificar lo aprendido por parte de los estudiantes.

De igual manera, haber realizado actividades grupales con las TIC permitió que los estudiantes valoraran el saber en sus pares, hecho que fortaleció lazos de amistad y el reconocimiento de las capacidades de algunos estudiantes dentro del grupo. El uso de herramientas TIC en actividades académicas facilitó el aprendizaje colaborativo a través del trabajo grupal, en el cual los estudiantes experimentaron las bondades de la colaboración. Pico y Rodríguez (2012) afirman que "la riqueza de la colaboración reside en que los estudiantes aprenden reflexionando sobre lo que hacen, ya que en el intercambio los saberes individuales se hacen explícitos y se tornan comprensibles para los demás" (p. 9). Ante una dificultad, los estudiantes buscaban la ayuda de algún compañero antes de buscar la ayuda de la profesora. 
La mediación tecnológica se dio en relación con la interacción entre estudiantes, profesor, las actividades usando las TIC. Durante cada sesión se pudo observar que, con las actividades propuestas para trabajar en grupo, usando herramientas digitales, se fortalecían las relaciones interpersonales entre estudiantes y profesor. Cabe recalcar que el trabajo en el aula de informática se caracterizó por el trabajo colaborativo, lo cual posibilitó la interacción y colaboración entre los estudiantes, potenciando así el aprendizaje colaborativo y la regulación de conflictos de modo pacífico. Todo lo anterior hace de las TIC instrumentos mediadores de la acción humana y del aprendizaje grupal e individual (Morera, Fortuny y Planas, 2012).

La evaluación formativa comprende un proceso conformado por varios pasos y no por un determinado tipo de examen (Popham, 2013). Su aplicación durante la secuencia se dio durante el proceso, constatando a través de la observación, la realimentación y atendiendo las inquietudes de los estudiantes, lo que permite realizar modificaciones a las actividades y tener en cuenta los objetivos de aprendizaje propuestos. Así se logró resolver las dificultades durante la secuencia de manera satisfactoria y apuntando a mejorar las estrategias y los aprendizajes.

El diario de campo como instrumento de recolección de datos permitió registrar con detalle comportamientos y actitudes de los estudiantes cuando interactúan con las TIC. Durante el desarrollo de las actividades que implicaron el uso de recursos TIC que componían la secuencia didáctica y, a través de la observación participativa y la reflexión, el investigador estuvo atento haciendo el seguimiento a esos comportamientos que podían denotar algún indicio relacionado con la vivencia de las competencias ciudadanas pluralidad, identidad y valoración de las diferencias entre los estudiantes durante el desarrollo de actividades, dando cumplimiento a los objetivos planteados. A través de la observación y análisis de dichos comportamientos, la toma de notas de campo y el registro en el diario de campo, se identificaron patrones comportamentales en los estudiantes poco usuales en el aula de clase. Fue notable en los estudiantes que, durante el desarrollo de las actividades de la secuencia didáctica, fueron más sociables, respetuosos, amables y colaboradores; los conflictos por apodos e irrespeto entre los estudiantes disminuyeron.

\section{Conclusiones}

El desarrollo de la competencia instrumental en la escolaridad de la primaria básica es fundamental para que los estudiantes logren una buena experiencia interactiva con las TIC. Esta subcategoría se manifestó a lo largo de la secuencia didáctica. Desde la sesión uno, se detectó en la mayoría de estudiantes poco dominio de las TIC pues, cuando se les pidió que escribieran en Microsoft Word o en PowerPoint, los acuerdos para el trabajo en el aula se les dificultó. Su mejoramiento fue notable en la medida en que se fue desarrollando cada sesión de la secuencia. Esto motivó a tener en cuenta los conocimientos digitales en cada estudiante e ir haciendo el seguimiento a su mejoramiento. 
A través de la alfabetización digital se pueden desarrollar competencias ciudadanas que den sentido a la pluralidad, la identidad y la valoración de las diferencias, en los estudiantes de la básica primaria, con el desarrollo de propuestas didácticas que involucren el uso continuo de las TIC. Propiciar en los estudiantes la adquisición de competencias digitales que les permita un tratamiento analítico y reflexivo de la información, conlleva a la transformación e integración de la sociedad de hoy, la cual se caracteriza por la construcción y promulgación de la información en línea. De ahí que uno de los propósitos de las políticas educativas ha sido la formación de mejores seres humanos, ciudadanos que promuevan los valores democráticos y sean respetuosos de lo público, ejerzan los derechos humanos y cumplan con sus responsabilidades sociales en todos los ámbitos de participación ciudadana (Flórez y Ramírez, 2014). Por lo tanto, brindar a los estudiantes espacios mediados por las TIC para la construcción colectiva del conocimiento es brindar la oportunidad de compartir sus saberes, de confrontar sus ideas, inquietudes y necesidades.

Con la integración curricular de las TIC se amplía el abanico de recursos didácticos aplicables en los procesos de enseñanza y aprendizaje, configurándose en estrategias que facilitan los aprendizajes (Rivero, Gómez y Abrego, 2013). Las propuestas didácticas mediadas por las TIC, enfocadas a construir ciudadanía en los estudiantes, arrojan buenos resultados siempre y cuando en su planeación se tenga en cuenta sus necesidades, el contexto en el cual están inmersos y los recursos con que se dispone. En la cotidianidad escolar se entretejen distintas situaciones comportamentales entre estudiantes, ante lo cual, el profesor ha de mediar entre las partes con inteligencia y diligencia aprovechando los sucesos como actos pedagógicos. En contextos escolares mediados por las tecnologías, esas situaciones se minimizan y son solucionadas entre los pares de manera concertada donde el dispositivo digital se convierte en mediador en la solución de las desavenencias ya que atraen toda la atención de los estudiantes.

Las propuestas didácticas que integran el uso de las TIC favorecen el trabajo colaborativo, en el que los aprendizajes no se dan de manera aislada, por el contrario, es un aprendizaje compartido entre los estudiantes. Lo anterior es posible en escenarios de aprendizaje que propicien la mutua colaboración para que cada integrante desde sus capacidades y conocimientos, pueda contribuir a la resolución del problema (Onrubia et al., 2008). Un ambiente colaborativo mediado por las TIC contribuye a la formación de competencias ciudadanas ya que, al contribuir cada integrante a la solución conjunta del problema, este se convierte en una meta en común, donde las partes han de ponerse de acuerdo y establecer un diálogo cordial que favorezca la valoración del saber en el otro. Implementar propuestas didácticas que vinculen el uso de las TIC en el aula implica un cambio en las prácticas educativas tanto del docente como del estudiante, debido a las posibilidades que ofrecen las herramientas digitales en cuanto a la autonomía, interacción, innovación y el uso de recursos. 
La alfabetización digital en la formación de competencias ciudadanas en la básica primaria

\section{Referencias}

Aguilar, M. (2012). Aprendizaje y Tecnologías de Información y Comunicación: Hacia nuevos escenarios educativos. Revista Latinoamericana de Ciencias Sociales, Niñez y Juventud, 10 (2), 801-811. Recuperado de www.redalyc.org/pdf/773/77323978002.pdf

Amaya, R. (2007). La investigación en la práctica educativa: Guía metodológica de investigación para el diagnóstico y evaluación en los centros docentes. Madrid, España: Fareso S.A.

Area, M. (2010). Tecnologías digitales, multialfabetización y bibliotecas en la escuela del siglo XXI. Boletín de la Asociación Andaluza de Bibliotecarios, 25 (98), 39-52. Recuperado de https: / / dialnet.unirioja.es/descarga/articulo/3616424.pdf

Avello, R., López, R., Cañedo, M., Álvarez, H., Granados, J. y Obando, F. (2013). Evolución de la alfabetización digital: nuevos conceptos y nuevas alfabetizaciones. Medisur, 11 (4), 450457. Recuperado de http://scielo.sld.cu/scielo.php?script=sci_arttext\&pid=S1727897X2013000400009

Casas, M.D., Mendiola, C., Hernández, I., Caballero, A.E., Elías, M.D.P., Barba, M. y Buenfil, M. (2015). El ejercicio responsable de la ciberciudadanía. Opción, 31 (5), 219-238. Recuperado de http:/ / 200.74.222.178/index.php/opcion/article/view/20649

Cerda, H. (1991). Medios, instrumentos, técnicas y métodos en la recolección de datos e información. En H. Cerda. (Ed.), Los elementos de la investigación (pp. 235-339). Bogotá, Colombia: El Búho.

Elliot, J. (2003). La investigación-acción en educación. Madrid, España: Ediciones Morata.

Flórez, K. y Ramírez, S. (2014). El desarrollo de la ciberciudadanía en Colombia a partir de prácticas educativas apoyadas en las TIC. Revista Digital Universitaria, 15 (12), 1-19. Recuperadode:http:/ /www.ru.tic.unam.mx/tic/bitstream/handle/123456789/2281/ art98_2014.pdf? sequence $=1$ \&isAllowed $=\mathrm{y}$

García, R., Ramírez, A. y Rodríguez, M. (2014). Educación en alfabetización mediática para una nueva ciudadanía. Comunicar, 43 (22), 15-23. Recuperado de http:/ /www.redalyc. org/html/158/15831058003/

Gross, B. y Contreras, D. (2006). La alfabetización digital y el desarrollo de competencias ciudadanas. Revista Iberoamericana de Educación, 42, 103-125. Recuperado de http:// diposit.ub.edu/dspace/handle/2445/57176

Gutiérrez, A. (2003). Alfabetización digital. Algo más que ratones y teclas. Barcelona, España: Gedisa.

Gutiérrez, A. (2010). El ciudadano territorial: propósito de la formación ciudadana. UNIPLURI/VERSIDAD, 10 (3), 1-12. Recuperado de https://aprendeenlinea.udea.edu.co/ revistas/index.php/unip/article/view/9578/8818 
Gutiérrez, A. y Tyner, K. (2012). Educación para los medios, alfabetización mediática y competencia digital. Comunicar, 19 (38), 32-39. Recuperado de http://dx.doi. org/10.3916/C38-2012-02-03

Hernández, R., Fernández, C. y Baptista, P. (2010). Metodología de la investigación. Ciudad de México, México: Mc-GrawHill, 5 Edición.

Malfasis, S. (2014). Estudio de caso: Fortalecimiento de las competencias ciudadanas a través de estrategias mediadas por las tecnologías de la información y la comunicación (Tesis de maestría). Universidad de La Sábana, Bogotá. Colombia. Recuperado de https://intellectum.unisabana.edu. co/bitstream/handle/10818/11587/Sayana\%20Malfasi\%20Martinez\%20(tesis)... pdf?sequence $=1$ \&isAllowed $=\mathrm{y}$ :

Marzal, M.Á. (2009). Evolución conceptual de la alfabetización en información a partir de la alfabetización múltiple en su perspectiva educativa y bibliotecaria. Investigación bibliotecológica, 23 (47), 129-160. Recuperado de http://www.ejournal.unam.mx/ibi/ vol23-47/IBI002304706.pdf

Ministerio de Educación Nacional (MEN). (2004). Estándares Básicos de Competencias Ciudadanas. Formar para la ciudadanía... Lo que necesitamos saber y saber hacer. Recuperado de https: / / www.mineducacion.gov.co/cvn/1665/articles-75768_archivo_pdf.pdf

Montaño, E. (2015). Estrategia educativa apoyada en TIC, para la formación en ciudadanía y convivencia, en estudiantes de secundaria de grados décimos y undécimos (Tesis de pregrado). Universidad Pedagógica Nacional, Bogotá, Colombia.

Morera, L., Fortuny, J.M. y Planas, N. (2012). Momentos clave en el aprendizaje de isometrías en un entorno colaborativo y tecnológico. Enseñanza de las Ciencias, 30 (1), 143-154. Recuperado de http://pagines.uab.cat/nuria_planas/sites/pagines.uab.cat.nuria_ planas/files/Morera_Fortuny_Planas_2012.pdf

Onrubia, J., Colomina, R. y Engel, A. (2008). Los entornos virtuales de aprendizaje basados en el trabajo en grupo y el aprendizaje colaborativo. En C. Coll. y C. Monereo. (Eds.), Psicología de la educación virtual. Enseñar y aprender con las tecnologías de la información y la comunicación (pp. 233-252). Madrid, España: Morata.

Pagés, J. (2012). Las competencias ciudadanas, una finalidad de la enseñanza de la historia. En S. Plá., X. Rodríguez. y V. Gómez. (Coords.), Miradas diversas a la enseñanza de la historia (pp. 19-66). Ciudad de México, México: Universidad Pedagógica Nacional.

Pérez, M. y Delgado, A. (2012). De la competencia digital y audiovisual a la competencia mediática: dimensiones e indicadores. Comunicar, 39 (X), 25-34. Recuperado de https: / / doi.org/10.3916/C39-2012-02-02 
La alfabetización digital en la formación de competencias ciudadanas en la básica primaria

Pico, L. y Rodríguez, C. (2012). Trabajos colaborativos: serie estrategias en el aula para el modelo 1 a 1. Recuperado de http://bibliotecadigital.educ.ar/uploads/contents/trabajos_ colaborativos0.pdf.

Popham, W.J. (2013). Evaluación trans-formativa: el poder transformador de la evaluación formativa. Madrid, España: Narcea Ediciones.

Rivero, I., Gómez, M.G. y Abrego, R.F. (2013). Tecnologías educativas y estrategias didácticas: criterios de selección. Revista educación y tecnología, (3), 190-206. Recuperado de https: / / dialnet.unirioja.es/descarga/articulo/4620616.pdf

Ruiz, J.I. (2012). Historias de vida. En J. I. Ruiz. (Ed.), Metodología de la investigación cualitativa (pp. 267-313). Bilbao, España: Universidad de Deusto.

Ruiz, A. y Chaux, E. (2005). La formación de competencias ciudadanas. Recuperado de https:// laasociacion.files.wordpress.com/2015/11/la-formacion-de-competencias-ciudadanas. pdf

Tapia, M., Correa, R., Ortiz, M. y Neira, A. (2012). Secuencia didáctica para la escritura en colaboración en un entorno wiki: Propuesta para estudiantes de enseñanza secundaria. Revista española de pedagogía, LXX (253), 553-571. Recuperado de https:// revistadepedagogia.org/wp-content/uploads/2012/09/253-09.pdf

Travieso, J.L. y Planella, J. (2008). La alfabetización digital como factor de inclusión social: una mirada crítica. UOC Papers. Revista sobre la sociedad del conocimiento, (6), 1-9. Recuperado de www.uoc.edu/uocpapers/6/dt/esp/travieso_planella.pdf. 